\title{
The air gap between tape and drum in a video recorder
}

\author{
H. Moes
}

University of Twente, Dept. of Mechanical Engineering, Tribology Group, P.O. Box 217, 7500 AE Enschede, The Netherlands

\begin{abstract}
Lubrication with ambient air is not quite generally applied. The best known application is the "foil bearing" in tape recording systems for audio, video and computer applications; where the gap height that is needed for effective lubrication may quite easily be attained. This air gap reduces tape friction and wear of tape and recording head substantially. On the other hand, though, the air gap for effective magnetic recording is very small. These conflicting demands on the lubrication conditions ask for an accurate calculation of the air gap distribution.

The multigrid method - a fast, iterative equation solver - will be applied to calculate the film thickness in foil bearings on a fine grid. The results for a simple but adequate model for the air lubrication between the tape and the drum in a VHS-recorder will be presented, including details about the narrow gap between recording head and tape.
\end{abstract}

\section{Nomenclature}

A tape length, $\mathrm{L}$

$B \quad$ tape width, L

$E$ tape elastic modulus, $\mathrm{FL}^{-2}$

$F$ biharmonic stress function, $\mathrm{FL}$

$G \quad$ maximum gap height, $\mathrm{L}$

h mesh size

$K$ tape thickness, $L$

$p$ dimensionless pressure

$P \quad$ pressure relative to ambient, $\mathrm{FL}^{-2}$

$s \quad$ dimensionless stress invariant

$S$ stress invariant, $\mathrm{F} / \mathrm{L}$

$T$ tape tension force, $\mathrm{F}$

$R$ drum radius, $\mathrm{L}$

$u \quad$ dimensionless biharmonic stress function

$U$ biharmonic stress function, FI.

$v$ dimensionless tape curvature invariant

$V$ tape curvature invariant, $1 / \mathrm{L}$

$w$ dimensionless gap height

$W \quad$ tape deflection, $\mathrm{L}$

$\bar{W} \quad$ gap height, $\mathrm{L}$

$x, y$ dimensionless coordinates

$X, Y$ plane coordinates, $\mathrm{L}$

$\bar{X}, \bar{Y} \quad$ cylinder coordinates, $\mathrm{L}$

$\alpha \quad$ tape length number

$\beta \quad$ tape width number

$\gamma \quad$ maximum gap height number

$\Delta \quad$ Laplace operator

$\kappa \quad$ flexural rigidity number

$\lambda$ tensional stiffness number

$\mu \quad$ air viscosity, FTL $^{-2}$

Poisson's ratio

angular velocity of the drum, $1 / \mathrm{T}$

\section{Introduction}

In various types of tape recording systems the tape acts as a foil bearing lubricated with ambient air. In video recording systems, for instance, a flexible tape, the recording medium, is tensioned around a rotating drum provided with recording heads. In this recorder lubrication with ambient air between tape and drum plays an important, quite often even a dominant, role. On the one hand, a substantial air gap reduces the friction, and consequently the tape stresses and the rubbing noise due to stick-slip, and eliminates the wear of the tape and the recording head. On the other hand, for effective recording in high density magnetic recorders the air gap between the tape and the head needs to be very small. Due to the persistence of air lubrication this asks for special measures. For more detailed information see Bharat Bhushan [1].

The air gap between head and tape will be calculated in two steps. The first step is the calculation of the geometry of the gap between tape and drum in a global way, i.e. neglecting the recording head for the time being. The second step is the calculation of the local effect of a protruding recording head located on the drum.

Methods for calculating the overall gap height in foil bearings have been given by several authors. 
Pioneers were Blok and Van Rossum [2]; they presented an approximate analytical solution by neglecting all second order effects. Later on the numerical solution given by Baumeister [3] was a substantial improvement. Whereas eventually the work of Eshel and Elrod [4,5] gave more detailed information about the geometry of the gap at the exit and the effect of flexural rigidity.

Only quite recently Fijnvandraat [6] introduced the one-dimensional approximation of a two-dimensional flow in the foil bearing calculations. Eventually Yoneda and Sawada [7] and Rongen [8] succeeded in calculating a complete solution for the foil bearing. Though both papers willfully neglect the intriguing anticlastic effect at the side edges.

Up to the time of writing of this paper three computer programs were known that predicted the local effects on the gap height of the various geometries of the head. Greenberg [9] was the first to introduce a rigorous air film calculation between spherical heads and the tape. According to the results a single wave occurs in the vicinity of the head. The calculation results by Wolf et al. [10] deals with more complicated geometries for the recording head. The results showed a number of waves around the head. Yoneda and Sawada [7] incorporated the local effects in the global calculation, including non-linear effects and flexural rigidity of the tape. Although the air gap in question, i.e. the flying height between head and tape, was set to zero.

The method of solution that will be presented in this paper is based on multilevel techniques. These techniques have already been applied to a similar problem, the ElastoHydrodynamic Lubrication (EHL), by Lubrecht et al. [11], Lubrecht [12] and Venner and Ten Napel [13]. According to these publications the Gauss-Seidel Iteration Scheme (GSIS) appears to be very well suited for solving all kinds of two-dimensional problems in relation to the lubrication between flexible surfaces. In particular since the application of MultiGrid (MG) transforms a GSIS into a fast, iterative matrix equation solver. This gave great promise of being able to solve the foil bearing lubrication problem on a fine grid.

The present work applies GSIS with MG to the non-linear, two-dimensional foil bearing problem taking into account the flexural rigidity of the tape. Both the overall geometry and a close-up in the vicinity of the head have been investigated.

\section{Modeling the tape}

The basic equations are the two equations for thin plate deformations and a third equation for the viscous flow in narrow gaps between perfectly smooth walls. Besides, a complete set of boundary conditions will be needed. And finally the resulting set of equations has to be adapted for computer calculations.

\subsection{Basic equations}

The two equations governing the deflection, $W$, for an initially flat, thin plate, subject to tensional stresses, will first be introduced (see also Chia [14]). These two equations are the compatibility equation:

$\Delta^{2} F-E K\left(W_{; X Y}^{2}-W_{: X X} W_{: Y Y}\right)=0$

and the equilibrium equation:

$\Delta F * \Delta W-\frac{E K^{3}}{12\left(1-\nu^{2}\right)} \Delta^{2} W+P=0$.

The parameters applied in these equations are $E$ for the tape elastic modulus, $\nu$ for Poisson's ratio and $K$ for the thickness of the tape.

The dependent variables figuring in these equations, apart from the tape deflection $W$, are $P$ for the pressure in the gap and $F$ for the biharmonic stress function. This stress function is related to the longitudinal plate stresses. On the other hand the deflection of the tape, $W$, is related to the plate bending moments per unit width and the perpendicular plate stresses; see appendix A.

The independent variables $X$ and $Y$ represent Cartesian coordinates. A semicolon in the index denotes differentiation with respect to the subsequent coordinates.

The symbol $\Delta$ represents the Laplace operator defined by:

$\Delta \Phi \equiv \Phi_{; X X}+\Phi_{: Y Y}$. 
Besides the following auxiliary definition has been introduced:

$\Delta \Phi * \Delta \Psi \equiv \Phi_{; X X} \Psi_{; Y Y}-2 \Phi_{; X Y} \Psi_{; X Y}+\Phi_{; Y Y} \Psi_{; X X}$

The third equation is the equation for the viscous flow in narrow gaps. For the application under consideration this is the well known Reynolds equation [15]:

$\left(\bar{W}^{3} P_{; X}\right)_{; X}+\left(\bar{W}^{3} P_{; Y}\right)_{; Y}-6 \mu \omega R \bar{W}_{; X}=0$

where $\bar{W}$ represents the gap height, $\mu$ the viscosity of air, $\omega$ the angular velocity of the drum and $R$ the radius of the drum.

Finally, the relation between the tape deflection, $W$, and the gap height, $\bar{W}$, will be given by introducing the following transformation:

$$
\begin{aligned}
& W_{; X X} \equiv \bar{W}_{; \bar{X} \bar{X}}-1 / R, \\
& W_{; Y Y} \equiv \bar{W}_{; \bar{Y} \bar{Y}}, \quad W_{; X Y} \equiv \bar{W}_{; \bar{X} \bar{Y}} .
\end{aligned}
$$

Note in particular that $X$ represents the coordinate in the tangential direction of the drum and $Y$ the coordinate in the axial direction; see fig. 1.

A complication is that substantial deflections occur. Since actually the Von Kármánn model that has been introduced for the tape is only valid for deflections smaller than a few times the thickness of the tape, the generally applied boundary conditions need to be adjusted. It will be clear that by introducing these equations the author implicitly introduced many more restrictions; for a discussion see section 5 .

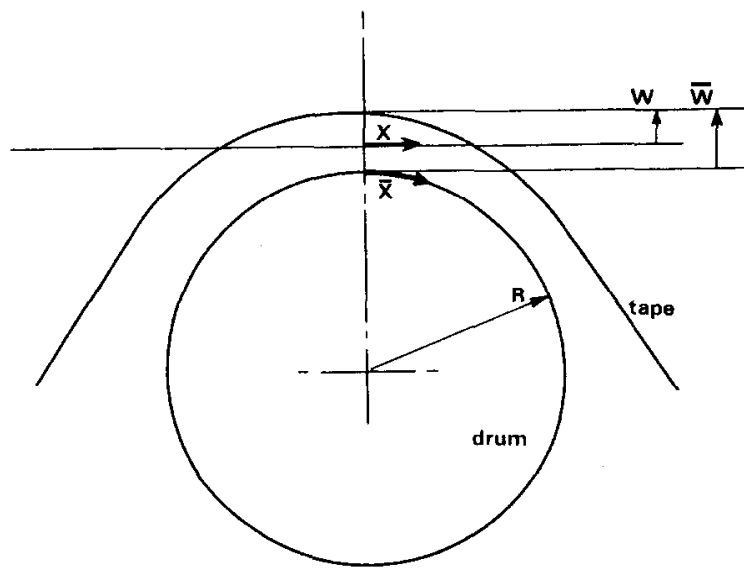

Fig. 1. The coordinates for tape-drum interaction.

\subsection{Boundary conditions}

The complete set of boundary conditions will now be introduced. These conditions are based on the following considerations:

- The pressure, $P$, is zero at all boundaries.

- By introducing $T$ for the tape tension force and $B$ for the tape width, the uniform tensional stress normal to the boundary of the tape may be chosen $T / B$ at entrance and exit (distant from the wrap area; therefore Saint-Venant's principle applies) and zero at the side edges. Therefore the stress function at all boundaries will be defined by $F=T Y^{2} / 2 B$.

- Since no shear stresses occur at the side edges and since $F=T Y^{2} / 2 B$, follows $F_{; Y}=T Y / B$.

- Quite arbitrarily $F_{; X X}=0$ will be assumed at entrance and at exit (again: distant from the wrap area; therefore Saint-Venant's principle applies).

- By lifting the tape over an arbitrary but substantial distance $G$ realistic uniform entrance and exit gaps will be created.

- At at all boundaries the external tape bending moment will be chosen equal to zero, leading to the first Kirchhoff boundary condition; at the side edges:

$$
W_{; Y Y}+\nu W_{; X X}=0 \text {. }
$$

- The side edges are free boundaries. Therefore the second Kirchhoff boundary condition too might be expected to apply; i.e.:

$\left\{W_{; Y Y}+(2-\nu) W_{; X X}\right\}_{; Y}=0$.

However, because of the substantial deflection in the $X$-direction of the tape, represented by $W_{; X X}$, this condition is rather inadequate. Anticlastic deformation, for instance, will not occur. Therefore instead of eq. (5) a new free boundary condition will now be introduced that will take into account the effect of $W_{x x}$.

Near the side of the tape normal plate stresses perpendicular to the edge are very small. Therefore the tangential strain $\epsilon$ depends on the normal stress in the tangential direction only and is given by $\epsilon=F_{Y Y Y} / E K$. This leads to the follow- 
ing geometrical condition for the gradient of the deflection normal to the side edge:

$W_{: Y}=-\epsilon_{; Y} / W_{: X X}$ for $W_{; X X}<0$.

Whereas for $W_{: X X}=0$ continuity needs to be maintained.

This will lead to the following equations at entrance and exit, where $X= \pm A / 2$ :

$$
\begin{aligned}
P & =F-(T / 2 B) Y^{2}=F_{; X X} \\
& =W_{; X X}+\nu W_{: Y Y}=\bar{W}-G-0 .
\end{aligned}
$$

Whereas at the side edges, where $Y= \pm B / 2$, apply:

$$
\begin{aligned}
P & =F-(T / 2 B) Y^{2}=F_{: Y}-(T / B) Y \\
& =W_{: Y Y}+\nu W_{: X X}=0
\end{aligned}
$$

and instead of eq. (5):

$$
\begin{aligned}
& W_{: X X}\left(F_{: Y Y Y}+E K W_{: X X} W_{: Y}\right)=0 \\
& \text { with } W_{; X X} \leq 0 \text { and } F_{: Y Y Y} Y \leq 0 .
\end{aligned}
$$

These conditions fully comply will the character of the three basic equations; i.e. at all boundaries one boundary condition is defined in terms of the pressure $P$, two boundary conditions are defined in terms of $F$ and two boundaries are defined in terms of $W$.

\subsection{Normalisation}

As a preparation to adequate computer programming it has shown to be useful to introduce the bipotential stress function, $U$, the stress invariant, $S$, and the tape curvature invariant, $V$ :

$U \equiv F-(T / 2 B) Y^{2}, \quad S \equiv \Delta U, \quad V \equiv \Delta W$.

Besides, by introducing similarity quantities the original set of equations, eqs. (1) through (8), may be substantially simplified; see appendix B. In this way eqs. (1) through (3) can be written:

$$
\begin{aligned}
& \Delta u-s=0, \\
& \Delta w-v-1=0, \\
& \Delta s-\lambda\left\{w_{i x y}^{2}-\left(w_{: x x}-1\right) w_{: y y}\right\}=0, \\
& \Delta u * \Delta w-u_{; y y}+w_{: x x}-\kappa \Delta v+p-1=0, \\
& \left(w^{3} p_{; x}\right)_{; x}+\left(w^{3} p_{; y}\right)_{, y}-w_{: x}=0 .
\end{aligned}
$$

In these equations $\Delta$ represents the Laplace operator with respect to $x$ and $y$ and $w$ represents the similarity variable for the gap height.

Of course the set of boundary conditions, eqs. (6) through (8), has to be adapted to this new set of equations. At entrance and at exit, i.e. for $x= \pm \alpha$, these conditions become:

$p=s=u=v=0$ and $w=\gamma$.

Whereas at the side edges, i.e. for $y= \pm \beta$, may be written:

$$
\begin{aligned}
& p=u=0, \\
& v-(1-v)\left(w_{i x x}-1\right)=0, \\
& u_{; y}=0, \\
& \left(w_{; x x}-1\right)\left\{s_{; y}+\lambda\left(w_{; x x}-1\right) w_{; y}\right\}=0, \\
& w_{; x x}-1 \leq 0, \\
& \left\{s_{: y}+\lambda\left(w_{; x x}-1\right) w_{; y}\right\} y \leq 0 .
\end{aligned}
$$

Note that the eqs. (13) through (15) represent a complementarity condition.

By applying this normalisation the set of ten original parameters has been replaced by a much more manageable set of five similarity parameters; i.e. $\alpha$ for the tape length, $\beta$ for the tape width, $\kappa$ for the flexural rigidity, $\lambda$ for the tensional stiffness and $\gamma$ for the air gap at entrance and at exit.

\section{Method of solution}

Multilevel relaxation has been applied as the method of solution. Actually, to begin with an error smoothing process was introduced by applying a Point-Gauss-Seidel-Newton Iteration Scheme (PGSNIS) with local linearisation. Next this process was transformed into a fast iterative solver by introducing multilevel relaxation. And finally some measures have been taken in order to stabilize the calculation process.

\subsection{Gauss-Seidel scheme}

By substituting the stress invariant, $S$, and the tape curvature invariant, $V$, through the eqs. (9) the original set of three partial differential equa- 
tions has been transformed into five second order partial differential equations, all of a quasi-elliptic type. Three of the equations are substantially non-linear.

The discretisations are based on the standard three point and five point discretisations for the one-dimensional and two-dimensional Laplace operator, respectively. For the discretisations of the Reynolds equation see Lubrecht [12]. A seven point discretisation, with the major axis normal to the prevailing direction of the iteration sweeps, has been applied to the hyperbolic operator. For details see appendix C.1.

The local Newton linearisation, introduced for the non-linear equations, means that Newton linearisation has been applied explicitly to the five central variables.

The two Neumann type boundary conditions, eqns. (12) and eqs. (13) through (15) have been based explicitly on mesh points immediately adjacent to the boundary. For details see appendix C.2.

Full relaxation sweeps were carried out in a specific order for the five equations involved; meanwhilc kccping the residuals for the four remaining equations untouched in the mesh point concerned. Subsequent to every sweep the variables at the boundaries were recalculated.

\subsection{Multigrid}

It is characteristic for a PGSNIS, when applied to sets of equations with great ellipticity, that after some relaxation sweeps the errors will be smooth. Hence they can be solved on a course grid, virtually without any loss of information. The iteration may be continued on this coarse grid by applying a coarse grid equivalent of the original equations; with the advantage that convergence will be much faster than on the original grid and thus asks much less solution time.

Finally, by means of the solution for the errors on this coarse grid the approximate solution on the original grid may be improved. Apart from a high frequency error caused by the necessary interpolations, a much better approximation to the solution will result than would have been possible by continuing relaxation on the original grid in equal time. The high frequency interpolation error causes no problems since it will almost completely disappear by applying a limited number of iteration sweeps; see Briggs [16].

This method, in a modification that is also suited for non-linear matrix operators, is called the Full Approximation Scheme (FAS) multigrid concept. Actually this is a defect-correction scheme; see Brandt [17]. It becomes extremely powerful when a multitude of levels is applied. On the coarsest level, though, the equations invariably have to be solved. This solution may quite well be derived by applying a large number of iteration sweeps since on a coarse grid this will not be time consuming.

The starting process is the Full MultiGrid (FMG) scheme [17]. By applying second order interpolation this provides for a good first approximation; and a good approximation is needed in order to guarantee convergence for the PGSNIS applied to non-linear equations.

\subsection{Special measures}

Because of the character of the linearisation applied, values for the gap width that are not positive will disturb the error smoothing process. Besides, non-positive gaps in practice are quite unnatural (actually it can be proven that any solution to the Reynolds equation possesses positive gaps only). Therefore non-positive values for the gap height, even the temporary ones popping up during relaxation, completely depend on the method and the level of discretisation. Two special measures have been taken to guarantee positive gaps:

- First, in order to prevent that non-positive solutions are needed for the gap height, the discretised third power of film thicknesses in the intermediate points that appear in the first terms of the discretised Reynolds equation according to Lubrecht [12] have been defined in a different way. For details see appendix C.3.

- Secondly, in order to put limitations to the non-linear effects when relaxation is applied, a special kind of under relaxation has been introduced. It limits the relative corrections for the 
gap height to $10 \%$ by applying a uniform reduction to all residual corrections prior to relaxation.

\section{Results}

The results to be presented for the tape-drum interaction apply to the following data for a VHS-recorder:

Tape elastic modulus:

$E=8 \mathrm{GPa}$,

Poisson's ratio:

$\nu=0.5$,

Tape thickness:

$K=20 \mu \mathrm{m}$,

Air viscosity:

Angular velocity of the drum:

Tape tension force:

$\mu=18.1 \mu \mathrm{Pa} \mathrm{s}$,

$\omega=157 \mathrm{~s}^{-1}$,

$T=0.5 \mathrm{~N}$,

$R=31 \mathrm{~mm}$,

Drum radius:

Tape length:

Tape width:

$A=100 \mathrm{~mm}$,

$B=12.7 \mathrm{~mm}$.

Actually Poisson's ratio is smaller (between 0.3 and 0.4 ) but this is of little effect. Consequently the control parameters are:

$\alpha=135.726, \quad \beta=17.237, \quad \kappa=0.333$,

$\lambda=2.296, \quad \gamma=10$.

Note that $\kappa$ is small. So the tape is quite flexible. Whereas $\gamma=10$ seems to be a fair choice for representing the actual tape.

Besides, the results to be presented for the tape-head interaction apply to the following dimensions of the head:

Radius of the head:

$10 \mathrm{~mm}$

Width of the head:

$200 \mu \mathrm{m}$,

Bulging radius of the head: $3 \mathrm{~mm}$,

Protrusion of the head: $\quad 50 \mu \mathrm{m}$.

Figs. 2 and 3 present the solution for a $25.4 \mathrm{~mm}$ element of tape with about $19 \mathrm{~mm}$ wrap region in the center. This wrap region represents an almost cylindrical element of tape that is conform with the drum. At entrance and at exit the $3 \mathrm{~mm}$ regions actually represent an almost flat tape. In reality the tape has a wrap region of about 100 $\mathrm{mm}$. This shortening of the tape, though, has nothing to do with the limited applicability of the Von Kármánn model. The tape was simply too long for a good graphical representation. Besides, nothing much happens in the dominating wrap region, apart from a slow decrease in gap height when going downstream. Already for this short element of tape a considerable central area with an almost uniform air gap of about $10 \mu \mathrm{m}$ predominates. By the way, this gap height complies very well, i.e. within $5 \%$, with the simple model results according to Baumeister [3]; see also appendix B.

The pressure distribution of fig. 2 shows a moderate pressure spike at the end of the wrap region followed by sub-ambient pressures at the begin of the exit area. Superficially this pressure spike agrees with the well known EHL solutions [12]. This spike is needed to create a short narrow gap at exit. The sub-ambient pressures are characteristic for gas lubrication.

The fig. 3, a close-up of the area at the left edge near to the end of the wrap region, reveals that:

1. The average gap height of about $10 \mu \mathrm{m}$ is locally reduced to about $4 \mu \mathrm{m}$.

2. A U-shaped narrow gap occurs with its open end in the direction of entrance. This geometry resembles the horse-shoe shaped narrow gap in EHL at point contact [12].

3. A substantial anticlastic deformation occurs along the edges in the wrap region. This effect was first predicted by Wildmann [18]. On the one hand, the upward curving of the tape as shown may be somewhat exaggerated due to the discretisation applied. And as a consequence it is not conclusive in confirming the experimental evidence that the film thickness at the edge is larger than at the remaining area; see Licht [19]. On the other hand, the side effects extend over almost $2 \mathrm{~mm}$ tape width. And this is in fair agreement with the $\sqrt{K R}$, for the tape under consideration this means about $1 \mathrm{~mm}$, according to the prediction made by Wildmann [18].

The method of calculation is also readily adaptable to computations around a head. Figs. 4 and 5 present a close-up of a small element of tape in the vicinity of a head. These diagrams follow from introducing a head geometry into the basic equations and from neglecting non-linear effects by 
applying first order perturbations to the tape equations; see also Wolf et al. [10]. The reason is that the non-linear effects, which disturb convergence for a protruding head, are of second order and may quite as well be neglected.

Comparing the solutions presented for a per- fectly flexible tape [10] and for a rather flexible tape [7] with the tape geometry of fig. 4, clearly shows the substantial effect of introducing the flexural rigidity. The conformity between head and tape is completely lost and waves do not occur. As a consequence the minimum gap height
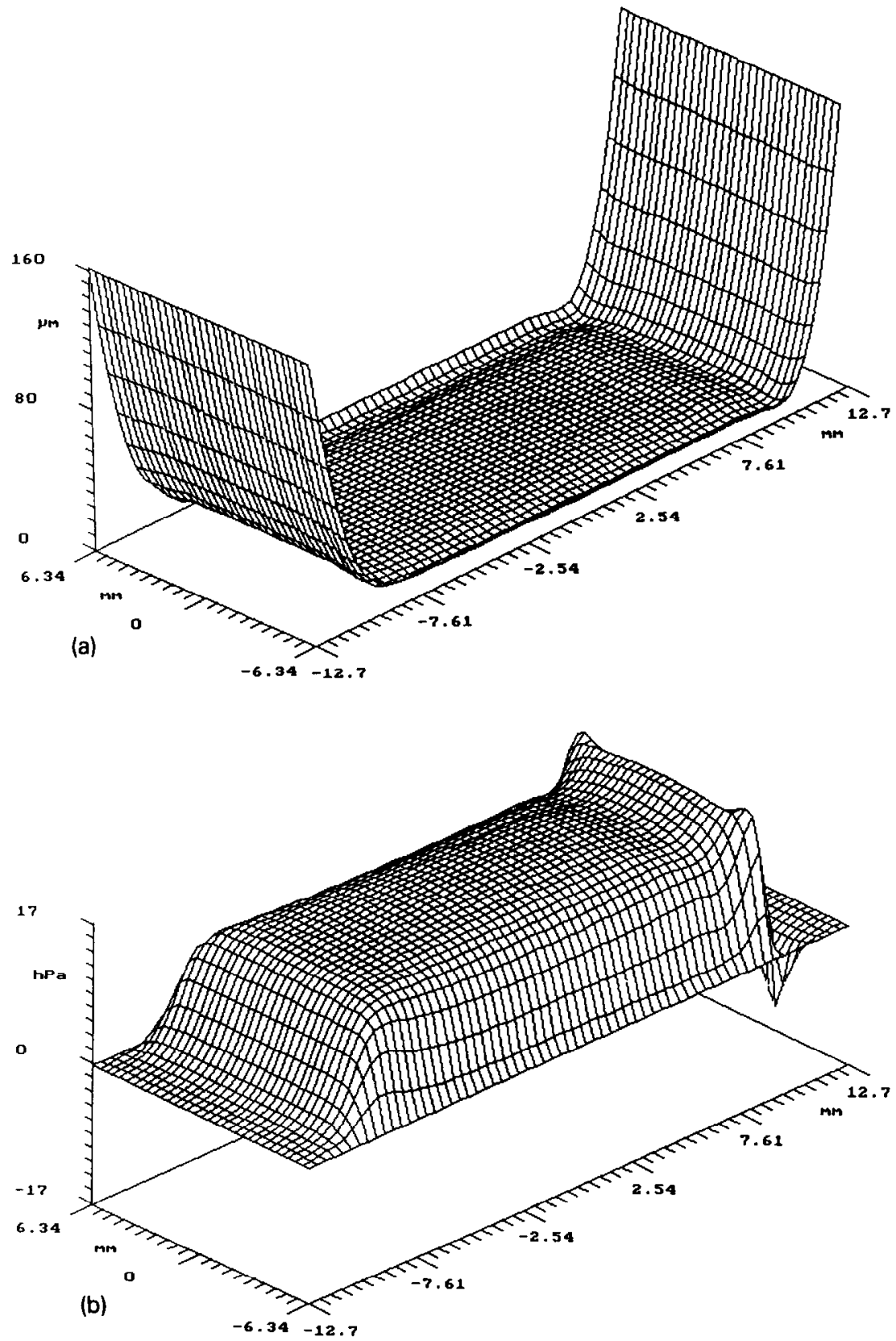

Fig. 2. The gap geometry and the corresponding pressure distribution. The drum rotates from left to right. 


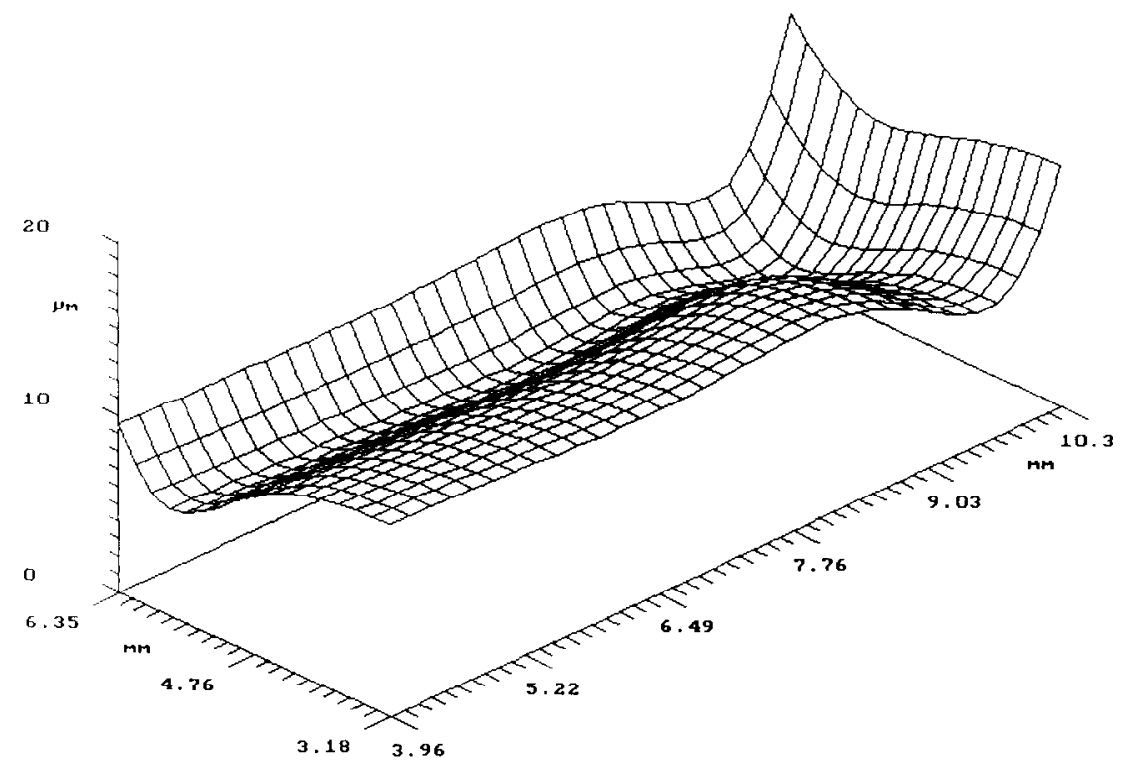

Fig. 3. A close-up of the minimum gap height at the side edge near the exit. The drum rotates from left to right.
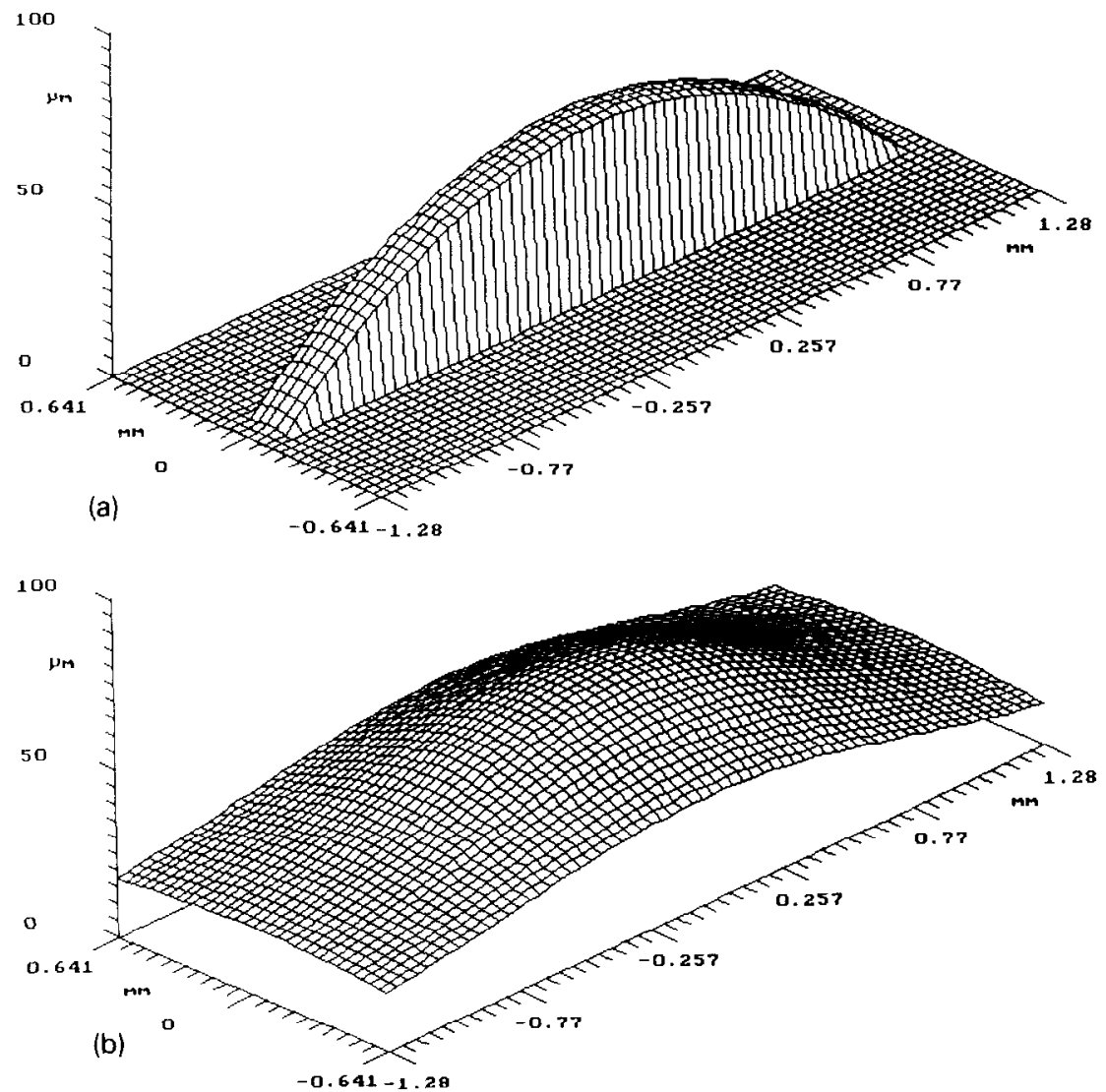

Fig. 4. The head geometry with the corresponding tape geometry. The drum rotates from left to right. 
is much smaller. According to fig. 5 a gap height of $0.03 \mu \mathrm{m}$ only occurs at the top of the head. At the side of the head, though, the gap height is much greater and surpasses $0.5 \mu \mathrm{m}$. The minimum gap height is $0.02 \mu \mathrm{m}$ and occurs in the trailing region of the head.

On the one hand, in relation to the surface roughnesses the minimum gap width of $0.02 \mu \mathrm{m}$ as calculated for perfectly smooth walls is much smaller than needed. On the other hand, a $0.5 \mu \mathrm{m}$ gap is much to thick for effective magnetic recording. As a consequence wear and rubbing noise may easily occur; whereas magnetic recording will still be far from optimal. In particular the bulging radius of the head has to be increased in order to obtain optimum conditions with respect to wear and registration efficiency!

Besides, fig. 5 shows that locally rather high
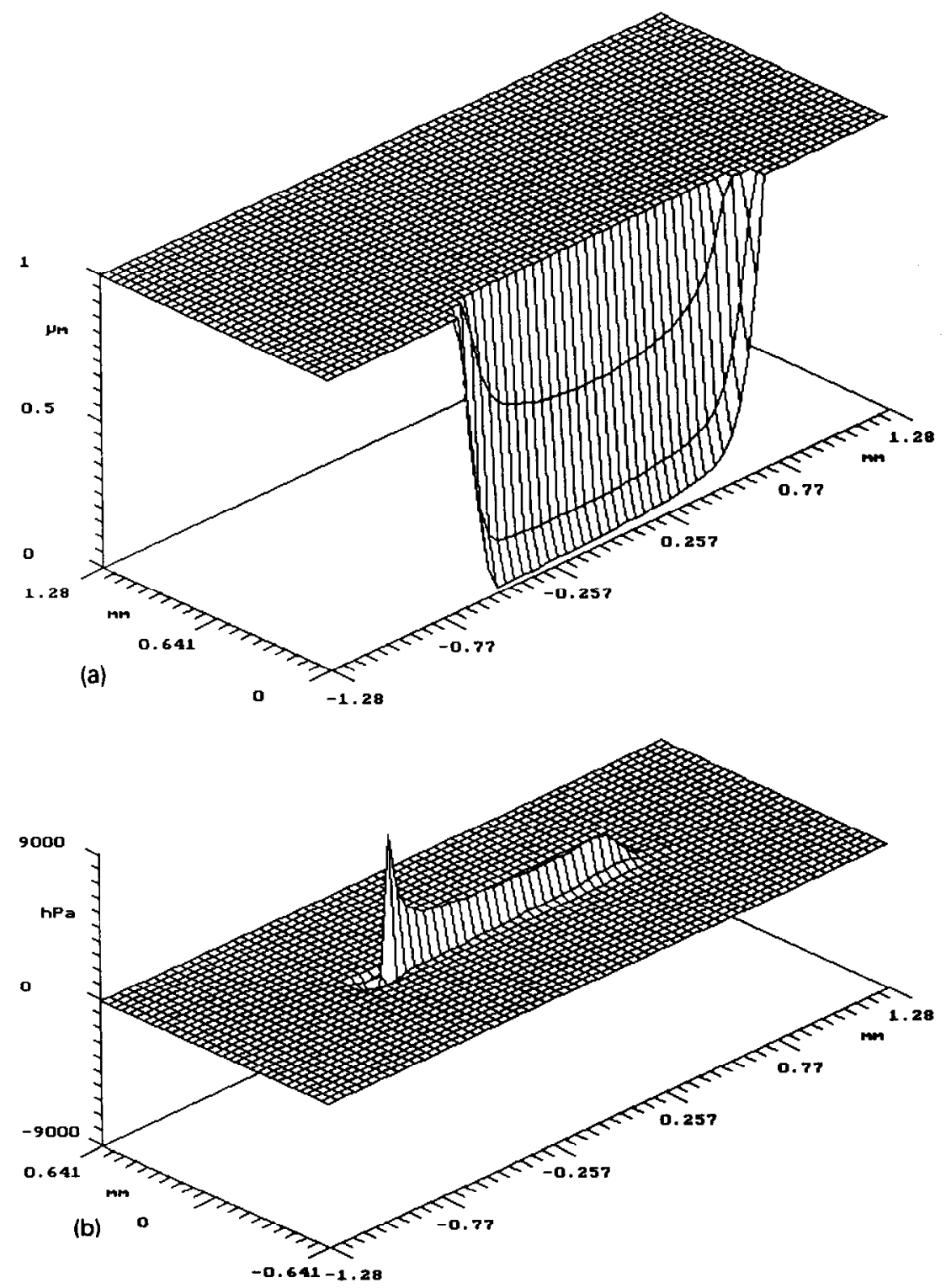

Fig. 5. Magnification of the gap height and the corresponding pressure distribution. The drum rotates from left to right. 
pressures occur. Because of a failing air lubrication due to the extremely thin gap this will lead to substantial friction forces; forces that eventually may damage the tape.

\section{Discussion}

The results that have been presented are solutions to the level of the truncation errors of the discretised equations. Therefore the convergence error is small enough not be distinguished from the diagrams. The dominating discretisation error, though, depends on the quality of the discretisation that has been applied. This means that the error in the location of the side edges may be of the order of magnitude of one mesh size. Nevertheless the anticlastic effect remains quite evident and the gap heights that have been presented may be considered fair approximations, with errors far below $10 \%$.

The model that has been introduced neglects various effects that may be of importance for the quality of the solution, such as:

1) All deviations from elastic plate and narrow gap flow theories.

2) All transient effects, like the head entry effect.

3) The helical tape guide.

4) The inhomogeneity and anisotropy of the tape.

5) The actual drum design, e.g. including mandrels and grooves.

6) The compressibility of air as a lubricant.

7) The Knudsen (i.e. molecular mean free path) effect on viscosity [10].

The first restriction is quite essential. Eqs. (1) and (2) have been based on the elastic theory of plates and the eq. (3) on the theory of flow in a narrow gap. As a consequence errors of $\mathcal{O}(K / B)$ respectively $\mathcal{O}(W / B)$, i.e. $\mathcal{O}(0.001)$, may be expected for the overall solution. Yet, more substantial errors may occur at the edges. Nevertheless the resulting errors for the gap height will be far below $1 \%$. And this is much smaller than the discretisation error.

The next four restrictions ( 2 through 5 ) do not represent serious limitations. They have been introduced for simplicity reasons only. The model can easily be modified in order to take account of the effects involved.

As to the last two restrictions (6 and 7), the results reveal that both the compressibility effect and the Knudsen effect are rather small as long as the head is not involved. In the vicinity of the head, however, these effects actually might not have been neglected. Consequently the film thickness will be much smaller than has been predicted! Unfortunately this has not been anticipated; otherwise an attempt would have been made to incorporate both effects in the computer program from the start.

In the MG scheme slow convergence, in particular in the begin when non-linear effects are dominating, had to be taken into account. As a consequence quite a number of V-cycles (up to 20) were needed in order to achieve truncation error quality for the solutions. At all levels, except at the coarsest level, six iteration sweeps proved to be optimal. At the coarsest level about one hundred sweeps were needed. The reason is that at the coarsest level the equations have to be solved, whereas at higher levels a smoothing of the differential errors satisfies.

\section{Conclusions}

The results indicate that a Point-GaussSeidel-Newton iteration scheme with multigrid is very well suited for calculating foil bearings, including the head-tape interaction in tape recorders. The method was found to have astonishing efficiency. Therefore an analysis of the air lubrication in magnetic recorders, incorporating many of the the effects mentioned above, seems quite feasible.

In the beginning the application of this method of calculation to elastohydrodynamic lubrication problems caused serious problems. The reason is the non-linear behavior of the Reynolds equation with respect to the film thickness and consequently the risk of negative film thicknesses occurring. Negative film thicknesses, apart from being unpractical, may disturb the convergence by introducing instabilities.

In order to overcome this problem some mea- 
sures had to be taken. These measures will reduce the rate of convergence at the start of the iteration process, where non-linear effects may be quite substantial.

With this problem solved, the calculations run smoothly, e.g. swiftly and without the conditional loops notorious in Newton-Raphson schemes. Besides, the following extra advantages of the application of this method may be mentioned:

- The method asks for a minimum of storage capacity, even if many grid points are to be applied. Actually the number of required addresses is directly proportional to $n \ln n$, with $n$ representing the number of degrees of freedom at the finest mesh.

- The method asks for a minimum of solution time. The reason is that the computer time too is directly proportional to $n \ln n$. For instance a $128 \times 256$ mesh (because of symmetry conditions actually $64 \times 256$ ) was solved in about $2 \mathrm{~h}$ on a $80386 / 87$ PC.

To stimulate multigrid applications in mechanical engineering and especially the problem of tape and head design a program with a simple application, amply provided with comment and in both PASCAL and FORTRAN, will be made available by request.

\section{Appendix A. Plate stresses}

The longitudinal plate stresses (two normal plate stesses and one shear plate stress) depend on the biharmonic stress function:

$N_{X X} \equiv F_{; Y Y}, \quad N_{Y Y} \equiv F_{; X X}, \quad N_{X Y} \equiv-F_{; X Y}$.

The plate bending moments per unit width (two normal bending moments and one skew bending moment) depend on the tape deflection:

$$
\begin{aligned}
M_{X X} & \equiv-\frac{E K^{3}}{12\left(1-\nu^{2}\right)}\left(W_{; X X}+\nu W_{; Y Y}\right), \\
M_{Y Y} & \equiv-\frac{E K^{3}}{12\left(1-\nu^{2}\right)}\left(W_{; Y Y}+\nu W_{; X X}\right), \\
M_{X Y} & \equiv \frac{E K^{3}}{12(1+\nu)} W_{; X Y}
\end{aligned}
$$

Besides, the two transverse plate stresses are:

$$
\begin{aligned}
& Q_{X} \equiv-\frac{E K^{3}}{12\left(1-\nu^{2}\right)}\left(W_{; Y Y}+W_{; X X}\right)_{; X}, \\
& Q_{Y} \equiv-\frac{E K^{3}}{12\left(1-\nu^{2}\right)}\left(W_{; Y Y}+W_{; X X}\right)_{; Y} .
\end{aligned}
$$

\section{Appendix B. Similarity quantities}

The following set of similarity quantities has been applied to replace the original set of quantities:

The independent similarity variables:

$x \equiv \frac{\bar{X}}{R} \sqrt[3]{\frac{T}{6 \mu \omega R B}}, \quad y \equiv \frac{\bar{Y}}{R} \sqrt[3]{\frac{T}{6 \mu \omega R B}}$.

The dependent similarity variables:

$p \equiv \frac{P R B}{T}, \quad s \equiv \frac{S B}{T}, \quad u \equiv \frac{U B}{T R^{2}} \sqrt[3]{\left(\frac{T}{6 \mu \omega R B}\right)^{2}}$,

$v \equiv V R, \quad w \equiv \frac{\bar{W}}{R} \sqrt[3]{\left(\frac{T}{6 \mu \omega R B}\right)^{2}}$.

And the similarity parameters:

$\alpha \equiv \frac{A}{2 R} \sqrt[3]{\frac{T}{6 \mu \omega R B}}, \quad \beta \equiv \frac{B}{2 R} \sqrt[3]{\frac{T}{6 \mu \omega R B}}$,

$\gamma \equiv \frac{G}{R} \sqrt[3]{\left(\frac{T}{6 \mu \omega R B}\right)^{2}}$

$\kappa \equiv \frac{E K^{3} B}{12 T R^{2}\left(1-\nu^{2}\right)} \sqrt[3]{\left(\frac{T}{6 \mu \omega R B}\right)^{2}}$,

$\lambda \equiv \frac{E K B}{T} \sqrt[3]{\left(\frac{6 \mu \omega R B}{T}\right)^{2}}$.

N.B. According to MG calculations Baumeister's nominal clearance number is:

$w=0.643040$ at $\partial p / \partial x=0$

for $\alpha, \beta, \gamma \rightarrow \infty$ and $\kappa=0$. 
Whereas over the complete wrap area both the gap height and the pressure distribution show a persistent undulation. This undulation in the gap height starts from the narrow gap at the end of the wrap area and extends in the upstream direction. The undulation in the pressure distribution starts from the pressure spike at the end of the pressurized area and is quite similar in appearance. For both undulations the wavelength, $l$, and the upstream attenuation factor per wave, $\theta$, are approximately:

$\frac{l}{R} \sqrt[3]{\frac{T}{6 \mu \omega R B}}=4.75$ and $\theta=0.025$

\section{Appendix C. Discretisations}

\section{C.1. Hyperbolic operator}

The seven point discretisation that has been applied to the hyperbolic operator in section 3.1 reads:

$$
\begin{aligned}
\frac{\partial^{2} f_{i, j}}{\partial x \partial y}= & -h^{-2}\left(f_{i+1, j}+f_{i-1, j}+f_{i, j+1}+f_{i, j-1}\right. \\
& \left.-2 f_{i, j}-f_{i+1, j+1}-f_{u i-1, i-1}\right)+\mathcal{O}\left(h^{2}\right) .
\end{aligned}
$$

\section{C.2. Boundaries}

Some details about the discretisation of the boundary conditions as mentioned in section 3.1 will be presented.

Introduce an $i, j$ mesh for the $x, y$-coordinate system with $h$ representing the uniform mesh size and $j=1$ representing one of the side edges of the tape. Then the boundary conditions according to the eqs. (11) and (12) may be discretisized:

$v_{i, 1}-(1-\nu)\left(D_{i}-1\right)=\mathcal{O}\left(h^{2}\right)$,

$s_{i, 1}-2 h^{-2} \tilde{u}_{i .2}=\mathcal{O}\left(h^{2}\right)$.

Eq. (15) has been discretisized by introducing for

$x_{\mathrm{b}, 1} \leq x_{i, 1} \leq x_{\mathrm{e}, 1}$ :

$w_{i, 1}-w_{i, 2}+\frac{s_{i, 1}-s_{i, 2}}{\lambda\left(D_{i}-1\right)}=\mathcal{O}(h)$ and eq. (14) by introducing for $x_{i, 1}<x_{\mathrm{b}, 1}$ :

$$
\begin{gathered}
w_{i, 1}-\left[\gamma+\left\{w_{\mathrm{b}, 1}-\gamma-\frac{1}{2}\left(x_{\mathrm{b}, 1}^{2}-\alpha^{2}\right)\right\}\right. \\
\left.\times \frac{\left|x_{i, 1}\right|-\alpha}{\left|x_{\mathrm{b}, 1}\right|-\alpha}+\frac{1}{2}\left(x_{i, 1}^{2}-\alpha^{2}\right)\right]=0
\end{gathered}
$$

and for $x_{i, 1}>x_{\mathrm{e}, 1}$ :

$$
\begin{gathered}
w_{i, 1}-\left[\gamma+\left\{w_{\mathrm{e} .1}-\gamma-\frac{1}{2}\left(x_{\mathrm{e} .1}^{2}-\alpha^{2}\right)\right\}\right. \\
\left.\times \frac{\left|x_{i, 1}\right|-\alpha}{\left|x_{\mathrm{e} .1}\right|-\alpha}+\frac{1}{2}\left(x_{i, 1}^{2}-\alpha^{2}\right)\right]=0 .
\end{gathered}
$$

The transition points $x_{b, 1}$ and $x_{\mathrm{e}, 1}$ are still adjusted by hand. Besides, for reasons of numerical stability the following mixcd discretisations were introduced:

$$
\begin{aligned}
\tilde{u}_{i, 2}= & \frac{1}{4}\left(u_{i-1.2}+2 u_{i, 2}+u_{i+1,2}\right), \\
D_{i}= & \frac{1}{8} h^{-2}\left(w_{i-2,2}+4 w_{i-1,2}-10 w_{i, 2}+4 w_{i+1.2}\right. \\
& \left.+w_{i+2.2}\right) .
\end{aligned}
$$

The introduction of $j=2$, i.e. neglecting boundary mesh points, in the last equation is intended to avoid implicit boundary conditions. Although this might increase the systematic error of $\mathcal{O}(h)$ that already occurs for the first order derivative in the Reynolds equation, i.e. in eq. (10). Anyhow a rather fine mesh will be needed in order to simulate the analytical solution accurately.

\section{C.3. Reynolds equation}

According to Lubrecht [12] the first term in the Reynolds equation (10) may be discretisized:

$$
\begin{aligned}
\frac{\partial}{\partial x}\left(w_{i}^{3} \frac{\partial p_{i}}{\partial x}\right) & \\
= & h^{-2}\left\{w_{i+1 / 2}^{3}\left(p_{i+1}-p_{i}\right)+w_{i-1 / 2}^{3}\left(p_{i-1}-p_{i}\right)\right\} \\
& +\mathcal{O}\left(h^{2}\right) .
\end{aligned}
$$

As mentioned in section 3.3 the third power of the gap heights in the intermediate points, figuring in this discretisation, have been defined by:

$$
w_{i+1 / 2}^{3} \equiv 2 \frac{w_{i}^{3} w_{i+1}^{3}}{w_{i}^{3}+w_{i+1}^{3}}, \quad w_{i-1 / 2}^{3} \equiv 2 \frac{w_{i}^{3} w_{i-1}^{3}}{w_{i}^{3}+w_{i-1}^{3}}
$$

with a similar discretisation for the second term. 


\section{References}

[1] Bharat Bhushan, Tribology and Mechanics of Magnetic Storage Devices (Springer-Verlag, New York, 1990).

[2] H. Blok and J.J. van Rossum, Lubrication Engineering 9 (1953) 316.

[3] H.K. Baumeister, IBM J. Res. Dev. 7 (1963) 153.

[4] A. Eshel and H.G. Elrod Jr., Trans ASME Series D 87 (1965) 831.

[5] A. Eshel and H.G. Elrod Jr., Trans ASME Series D 89 (1967) 92.

[6] J.G. Fijnvandraat, in: Proc 13th Leeds-Lyon Symp. on Tribology, ed. D. Dowson (Butterworth, Guildford, 1987) p.539.

[7] K. Yoneda and T. Sawada, IEEE Trans. Magn MAG-24 (1988) 2766.

[8] P. Rongen, On Numerical Solution of the Stationary 2D Foil Bearing Problem, Presentation at the 1990 ASME/ STLE Tribology Conference.

[9] H.J. Greenberg, IBM J. Res. Dev. 23 (1979) 197.
[10] B. Wolf, N. Deshpande and V. Castelli, Trans. ASME J. Tribology 105 (1983) 138

[11] A.A. Lubrecht, W.E. Ten Napel and R. Bosma, Trans. ASME J. Tribology 109 (1986) 551.

[12] A.A. Lubrecht, Ph.D. Thesis, University of Twente, Enschede (1987).

[13] C.H. Venner and W.E. Ten Napel, in: Proc. Eurotrib 1989, ed. K. Holmberg (The Finnish Society for Tribology, Helsinki, 1989) p.196.

[14] Chia Chuen-yuan, Nonlinear Analysis of Plates (McGraw-Hill, New York, 1980).

[15] O. Reynolds, Trans. Roy. Soc. 177 (1886) 157.

[16] W.L. Briggs, A Multigrid Tutorial (Society for Industrial and Applied Mathematics, Philadelphia, 1987).

[17] A. Brandt, Multigrid Techniques: 1984 Guide with Applications to Fluid Dynamics, GMD-Studien Nr. 85 (Gesellschaft für Mathematik und Datenverarbeitung $\mathrm{MBH}$, Bonn, 1984).

[18] M. Wildmann, Trans. ASME J. Lubr. Techn. 91 (1969) 37.

[19] L. Licht, Trans. ASME J. Lubr. Techn. 90 (1968) 199. 